\title{
Seasonal Variations and Correlations between Vitamin D and Total Testosterone Levels
}

\author{
Moo-Yeol Sim, Soo-Hyun Kim, Kwang-Min Kim* \\ Department of Family Medicine, Ajou University Hospital, Suwon, Korea
}

Background: Some studies have provided evidence for a possible association between vitamin D and testosterone levels; however, the evidence from studies in Koreans is inconsistent. In addition, insufficient evidence is available to support an association between seasonal variations in vitamin D and testosterone levels in Koreans. Therefore, we aimed to investigate the association between vitamin $\mathrm{D}$ and testosterone levels, and between seasonal variations in these levels in Korean men.

Methods: This cross-sectional study included 1,559 men, aged 25-86 years, who underwent a medical examination. We measured serum 25-hydroxyvitamin $\mathrm{D}(25[\mathrm{OH}] \mathrm{D})$ and total testosterone levels, and compared other laboratory test results and patient lifestyle characteristics. On the basis of sample collection time, we categorized patients into four seasons, and analyzed seasonal variability in 25(OH)D and total testosterone levels.

Results: The average participant age ( \pm standard deviation) was $53.3 \pm 8.8$ years, and the average serum $25(\mathrm{OH}) \mathrm{D}$ and total testosterone levels were $15.9 \pm 7.0 \mathrm{ng} / \mathrm{mL}$ and $5.1 \pm 1.6 \mathrm{ng} / \mathrm{mL}$, respectively. In the analysis of variance (ANOVA) model, no significant association was found between $25(\mathrm{OH}) \mathrm{D}$ and testosterone levels $(\mathrm{P}=0.51)$. ANOVA of the average 25(OH)D levels in season-based groups revealed significant seasonal variations in 25(OH)D levels (P-value for trend $<0.001$ ). No significant association was found between seasonal variations in total testosterone levels $(\mathrm{P}=0.06)$. However, after adjustment for confounding variables, total testosterone and 25(OH)D showed significant seasonal variability ( $\mathrm{P}=0.007$ and $\mathrm{P}<0.001$, respectively).

Conclusion: We found no significant correlation between serum 25(OH)D and total testosterone levels in Korean men. Moreover, serum 25(OH)D and total testosterone levels showed significant seasonal variations.

Keywords: Vitamin D; Testosterone; Seasonal Variation; Korean Men 


\section{INTRODUCTION}

Previous studies have indicated that vitamin D synthesis is affected by seasons and geographical latitude. ${ }^{1)}$ For example, a Japanese study reported that vitamin D synthesis is affected by seasons and geographical location. ${ }^{2}$ In Korea, some studies showed that vitamin D levels varied with geographic location ${ }^{3)}$; however, measurement of vitamin D levels during each season did not reveal any significant differences.

Vitamin D deficiency is associated with cardiovascular events, metabolic syndrome, diabetes mellitus (DM), chronic kidney disease, infection, osteoarthritis, low muscle strength, urinary incontinence, inflammatory bowel disease, and sleep apnea. ${ }^{4)}$

Biological actions of activated vitamin D (1,25-dihydroxyvitamin D $[1,25(\mathrm{OH}) 2 \mathrm{D}])$ are mediated by the vitamin $\mathrm{D}$ receptor (VDR). The VDR is expressed in reproductive tissues, such as testes, Sertoli cells, Leydig cells, prostate, seminal vesicles, and sperm. ${ }^{5)}$ A recent study suggested that vitamin D supplementation increases testosterone levels. ${ }^{6)}$ Other studies have also suggested a positive association between vitamin D levels and testosterone levels in $\mathrm{men}^{7}$; however, such studies have not been conducted in Korean men.

Testosterone performs several beneficial functions in men, but total testosterone levels decrease with age. Lower testosterone is associated with osteoporosis, sexual dysfunction, and poor quality of life. Recently, lower testosterone levels were implicated in other health issues such as depressed mood, fatigue, cognitive impairment, insulin resistance, type $2 \mathrm{DM}$, hypertension, obesity, and metabolic syndrome. ${ }^{8)}$ Therefore, increasing testosterone levels improves the quality of life and helps prevent low testosterone-associated health issues in men.

Deficiencies in either vitamin D or testosterone can cause cardiovascular events, metabolic syndrome, DM, and emotional instability. Vitamin D and testosterone deficiency have similar symptoms, and therefore, may be closely related to one another. Considering the crucial functions mediated by both vitamin $\mathrm{D}$ and testosterone, it is clinically important to identify the nature of the association in their levels.

Considering that vitamin D and testosterone deficiencies have very similar symptoms and that vitamin D levels undergo seasonal variations, it is possible that testosterone levels also vary according to the season. This hypothesis is supported by the results of recent studies, which showed that testosterone levels vary with the seasons. ${ }^{9,10)}$ One Korean study that attempted to identify seasonal variations in testosterone levels found that free testosterone levels, but not total testosterone levels, underwent seasonal variations. ${ }^{11)}$ Because total testosterone levels are used for the diagnosis of andropause by many primary hospital doctors, it is clinically important to evaluate seasonal variations in total testosterone levels.

The aim of this study was to determine whether serum vitamin D levels are positively correlated with testosterone levels in Korean males, and whether both vitamin $\mathrm{D}$ and testosterone levels undergo seasonal variations.

\section{METHODS}

\section{Participants and Data Collection}

This was a cross-sectional study conducted using university hospital medical records. Inclusion criteria were male patients who underwent a medical health examination between January 2012 and April 2014, and who underwent tests for measurement of total testosterone and 25-hydroxyvitamin D (25[OH]D) levels.

A total of 28,579 male patients aged 19-89 years underwent a medical examination, and of these, 1,559 patients aged 25-86 years underwent tests for measurement of total testosterone and 25(OH)D levels. Exclusion criteria were as follows: current testosterone replacement therapy; other medical conditions that could affect vitamin D or testosterone levels, such as cancer, thyroid disease, parathyroid disease, liver disease, inflammatory bowel disease, epilepsy, malabsorption, and bowel surgery; and vitamin D supplementation within the previous 3 months.

Data on lifestyle characteristics, such as smoking history, drinking history, and physical activity, were obtained by asking the patients to fill out a questionnaire. This study protocol was approved by the institutional review board (IRB) of Ajou University Hospital (IRB number: AJIRB-MED-MDB-11-386).

\section{Measurement}

Serum 25(OH)D levels were used to indicate serum vitamin D levels because the serum 25(OH)D level is an accurate representation of the serum vitamin D level.

We divided the participants into four groups on the basis of their 25(OH)D levels: group $1(25[\mathrm{OH}] \mathrm{D} \leq 10.3 \mathrm{ng} / \mathrm{mL})$, group $2(25[\mathrm{OH}] \mathrm{D}$, $10.4-14.7 \mathrm{ng} / \mathrm{mL})$, group $3(25[\mathrm{OH}] \mathrm{D}, 14.8-20.4 \mathrm{ng} / \mathrm{mL})$, and group 4 $(25[\mathrm{OH}] \mathrm{D} \leq 20.5 \mathrm{ng} / \mathrm{mL})$.

Data on the levels of other androgenic hormones such as free testosterone, sex hormone-binding globulin, luteinizing hormone, and estrogen were extracted from the records; however, the number of patients who had undergone these tests was low. Therefore, the levels of these hormones were not considered in this study.

In addition, the participants were classified according to their smoking or drinking status as never smoked or drank, currently smoking or drinking, or quit smoking or drinking. Participants were classified on the basis of physical activity into the following categories: no activity, sedentary status, light activity, regular activity, or rigorous activity. If the patient did not drink or smoke, they were assigned a score of 0 . Currently drinking or smoking resulted in a score of 1 . If they quit drinking or smoking, their score was 2 . In terms of physical activity, no activity was scored as 0 , sedentary status as 1 , light activity as 2 , regular activity as 3 , and rigorous activity as 4 .

Other laboratory data were obtained by medical examination using and tests standard methods: levels of fasting glucose (FG), glycated hemoglobin, aspartate transaminase and alanine transaminase, gamma-glutamyl transferase, total cholesterol, triglycerides, high-density lipoprotein (HDL), low-density lipoprotein (LDL), prostate-specific 
Table 1. Overall baseline characteristics of participants, and baseline characteristics according to 25(OH)D levels

\begin{tabular}{|c|c|c|c|c|c|c|}
\hline \multirow{2}{*}{ Variable } & \multirow{2}{*}{ Total } & \multicolumn{4}{|c|}{ 25(OH)D (ng/mL) } & \multirow{2}{*}{ P-value* } \\
\hline & & 1st quartile $\leq 10.3$ & $10.4 \leq 2$ nd quartile $\leq 14.7$ & $14.8 \leq$ 3rd quartile $\leq 20.4$ & $20.5 \geq 4$ th quartile & \\
\hline Number & 1,069 & 265 & 270 & 271 & 263 & \\
\hline Age (y) & $53.3 \pm 8.8$ & $53.2 \pm 9.7$ & $52.1 \pm 8.7$ & $53.8 \pm 8.4$ & $54.3 \pm 8.3$ & 0.03 \\
\hline Aspartate transaminase (U/L) & $28.2 \pm 8.1$ & $27.8 \pm 8.2$ & $28.3 \pm 7.7$ & $28.3 \pm 8.2$ & $28.2 \pm 8.3$ & 0.85 \\
\hline Alanine transaminase (U/L) & $27.5 \pm 12.4$ & $27.7 \pm 12.9$ & $28.1 \pm 12.2$ & $26.6 \pm 11.6$ & $27.6 \pm 12.9$ & 0.56 \\
\hline Total cholesterol (mg/dL) & $200.7 \pm 35.5$ & $196.0 \pm 36.9$ & $199.3 \pm 35.2$ & $203.1 \pm 34.3$ & $204.2 \pm 35.3$ & 0.03 \\
\hline Low-density lipoprotein (mg/dL) & $125.6 \pm 34.1$ & $122.5 \pm 35.9$ & $125.6 \pm 33.5$ & $126.1 \pm 33.9$ & $128.1 \pm 32.8$ & 0.31 \\
\hline Fasting glucose (mg/dL) & $102.0 \pm 23.5$ & $101.8 \pm 19.1$ & $102.9 \pm 28.4$ & $100.8 \pm 23.2$ & $102.4 \pm 22.3$ & 0.77 \\
\hline Hemoglobin A1c (\%) & $5.9 \pm 0.7$ & $5.9 \pm 0.6$ & $5.9 \pm 0.8$ & $5.9 \pm 0.7$ & $5.9 \pm 0.6$ & 0.96 \\
\hline Prostate specific antigen (ng/mL) & $1.0 \pm 0.8$ & $1.0 \pm 0.8$ & $1.0 \pm 0.8$ & $1.0 \pm 0.7$ & $1.0 \pm 0.9$ & 1.00 \\
\hline Total testosterone (ng/mL) & $5.1 \pm 1.6$ & $5.0 \pm 1.7$ & $5.1 \pm 1.7$ & $5.1 \pm 1.6$ & $5.2 \pm 1.6$ & 0.51 \\
\hline 25(OH)D (ng/mL) & $15.9 \pm 7.0$ & - & - & - & - & - \\
\hline Body mass index $\left(\mathrm{kg} / \mathrm{m}^{2}\right)$ & $24.5 \pm 2.8$ & $24.4 \pm 2.8$ & $24.5 \pm 2.8$ & $24.5 \pm 2.9$ & $24.6 \pm 2.8$ & 0.94 \\
\hline Waist circumference (cm) & $86.5 \pm 7.9$ & $86.3 \pm 7.4$ & $86.1 \pm 8.6$ & $86.7 \pm 8.1$ & $86.9 \pm 7.5$ & 0.63 \\
\hline Drinking (score) & $0.9 \pm 0.5$ & $0.9 \pm 0.5$ & $0.9 \pm 0.4$ & $1.0 \pm 0.5$ & $1.0 \pm 0.5$ & 0.66 \\
\hline Smoking (score) & $1.2 \pm 0.8$ & $1.2 \pm 0.8$ & $1.2 \pm 0.7$ & $1.2 \pm 0.8$ & $1.3 \pm 0.8$ & 0.81 \\
\hline Physical activity (score) & $1.8 \pm 1.2$ & $1.7 \pm 1.1$ & $1.8 \pm 1.2$ & $1.8 \pm 1.2$ & $2.1 \pm 1.2$ & $<0.01$ \\
\hline
\end{tabular}

Values are presented as mean \pm standard deviation.

25(OH)D, 25-hydroxyvitamin D.

*P-value for trend calculated by analysis of variance with Tukey post hoc test.

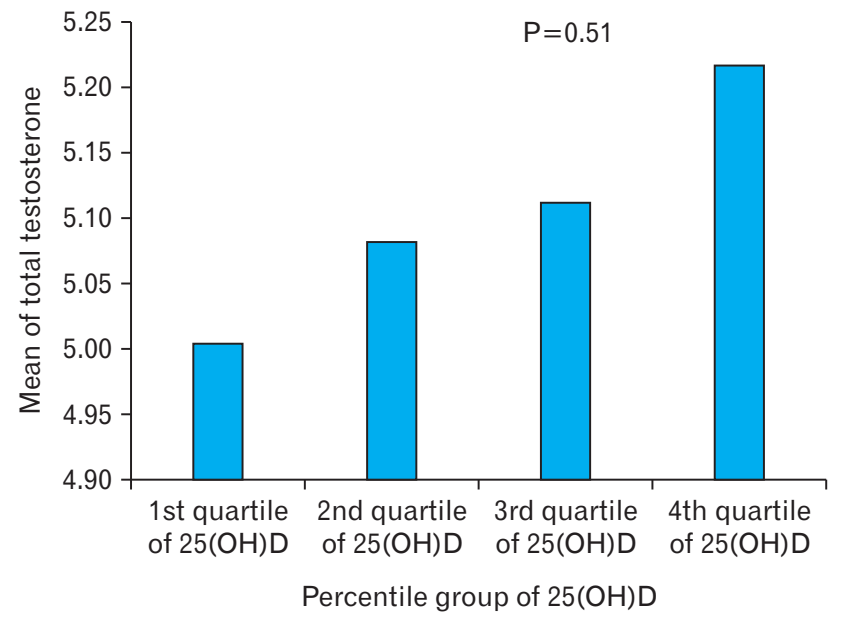

Figure 1. Total testosterone level according to the quartile of $25(\mathrm{OH}) \mathrm{D}$ level. Calculated by analysis of variance with Tukey post hoc test. $25(\mathrm{OH}) \mathrm{D}$, 25-hydroxyvitamin D.

antigen, and body mass index (BMI), and waist circumference (WC).

The participants fasted for 8 hours before the study, and blood samples were collected in a vacuum tube with anticoagulant between 7 $\mathrm{AM}$ and $11 \mathrm{AM}$. Blood samples were centrifuged prior to testing.

The serum 25(OH)D level was measured with chemiluminescence immunoassay using ADVIA centaur vitamin D total assay (Siemens Healthcare Diagnostic Inc., Tarrytown, NY, USA) at the Ajou University Hospital laboratory room (Suwon, Korea), which reported inter-assay and intra-assay coefficients of variation $<10 \%$.

Total testosterone levels were measured via a radioimmunoassay using Freedom EVO clinical 150/8 (TECAN, Mannedorf, Switzerland) at the Ajou University Hospital laboratory room (Suwon, Korea), which reported coefficients of variation $<5 \%$.

\section{Statistical Analysis}

Statistical analysis was performed using IBM SPSS ver. 22.0 (IBM Corp., Armonk, NY, USA). Participants were categorized into four groups based on 25(OH)D levels, in order to compare 25(OH)D levels with total testosterone levels. Other variables were compared with 25(OH)D levels directly.

We categorized patients into four groups based on the season in which they were evaluated: season 1 compromised March, April, May (spring); season 2 comprised June, July, and August; season 3 comprised September, October, and November; and season 4 comprised December, January, and February. Analyses were performed to detect correlations between total testosterone and 25(OH)D levels for the different seasonal categories.

We use one-way analysis of variance, followed by a Tukey post hoc test when appropriate. Seasonal variations in 25(OH)D and testosterone levels were assessed using analysis of covariance, after adjusting for age, total cholesterol, LDL, HDL, WC, BMI, FG, physical activity, alcohol use, and smoking. $\mathrm{P}<0.05$ was considered statistically significant. Data are presented as average \pm standard deviation.

\section{RESULTS}

\section{Patient Baseline Characteristics and Association of 25(OH)D Quartile with Total Testosterone Levels}

A total of 1,069 male patients were included in this study. Their average age was $53.3 \pm 8.8$ years, the average serum $25(\mathrm{OH}) \mathrm{D}$ level was $15.9 \pm 7.0 \mathrm{ng} / \mathrm{mL}$, and the average serum total testosterone level was $5.1 \pm 1.6 \mathrm{ng} / \mathrm{mL}$. 
The overall baseline characteristics of the study participants and the characteristics according to the level of 25(OH)D are presented in Table 1. Participants in the higher quartiles of $25(\mathrm{OH}) \mathrm{D}$ had higher total cholesterol levels and more physical activity. However, although participants in the higher quartile of 25(OH)D tended to have higher total testosterone, this trend was not significant, disproving our original hypothesis (Figure 1).

After adjustment for age, total cholesterol, LDL, HDL, WC, BMI, FG, physical activity, alcohol use, and smoking, no significant correlation was found between 25(OH)D and total testosterone levels ( $\mathrm{P}=0.78)$.

\section{Seasonal Variability in 25(OH)D and Total Testosterone Levels}

In the seasonal variation model, serum 25(OH)D levels were lowest in the spring and highest in the autumn $(\mathrm{P}<0.001)$. Serum 25(OH)D levels were similar in the spring and winter $(13.8 \pm 6.2$ and $15.9 \pm 6.7$, respectively; $\mathrm{P}=0.172)$ and in the summer and fall (18.5 \pm 7.2 and $18.1 \pm 6.8$, respectively; $\mathrm{P}=0.940)$. Despite these similarities, a significant $(\mathrm{P}<0.01)$ correlation was found between seasonal variations in serum $25(\mathrm{OH}) \mathrm{D}$ levels (Figure 2).

In contrast, total testosterone level was lowest in the summer and highest in the spring $(4.9 \pm 1.5$ versus $5.3 \pm 1.6$, respectively; $\mathrm{P}=0.05)$. However, no significant associations were found between seasonal variations in total testosterone in the seasonal model (spring versus fall, $\mathrm{P}=0.404$; spring versus winter, $\mathrm{P}=0.716$; summer versus fall, $\mathrm{P}=0.751$; summer versus winter, $\mathrm{P}=0.188$; and fall versus winter, $\mathrm{P}=0.857$ ). In addition, the $\mathrm{P}$-value for the trend in testosterone levels in the seasonal model was 0.06 (Figure 2). Therefore, although we found no significant association in seasonal variations in total testosterone levels, there was a significant trend.

$\square$ Mean of serum 25(OH)D level $\left(P^{*}<0.01\right)$

$\square$ Mean of serum total testosterone level $\left(P^{*}=0.06\right)$

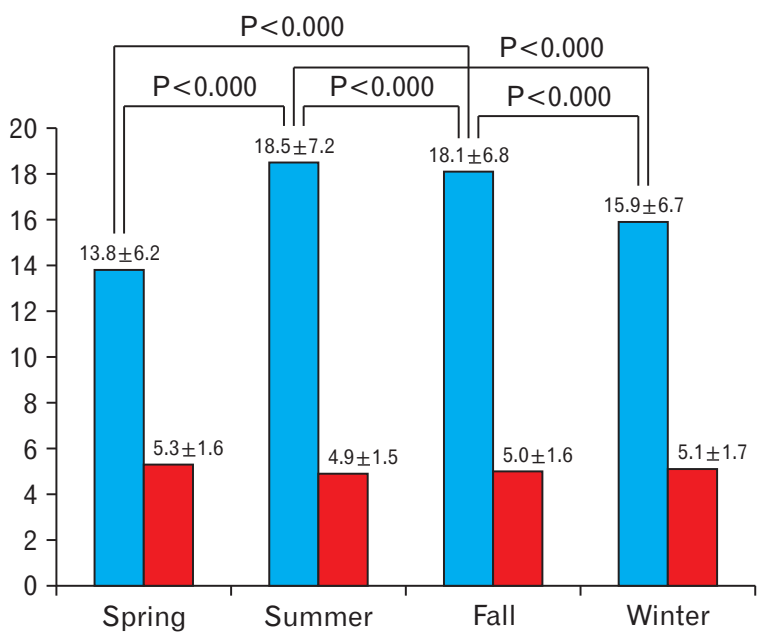

Figure 2. Seasonal variation in mean of $25(\mathrm{OH}) \mathrm{D}$ level and total testosterone level. Calculated by analysis of variance with Tukey post hoc test. 25(OH)D, 25-hydroxyvitamin D. *P for trend.
To find correlations within seasonal variations in total testosterone and serum 25(OH)D levels, we adjusted the data for age, total cholesterol, LDL, HDL, WC, BMI, FG, physical activity, alcohol use, and smoking. Data was divided into four models based on the variables that were adjusted. The results of model 1 are shown in Figure 2. The results of model 2 were the same as those of model 1 . However, in model 3 and model 4 , seasonal variations in total testosterone and serum 25(OH)D levels showed a significant correlation (Table 2).

\section{DISCUSSION}

In this study, we found no correlation between serum 25(OH)D and total testosterone levels, even after adjustment for potential confounding variables. However, we found a significant association between seasonal variations in 25(OH)D levels, and a correlational tendency in seasonal variations of total testosterone levels. In addition, after adjustment for confounding variables a significant correlation was found in seasonal variations in total testosterone levels.

A recent study reported a positive correlation between vitamin D and total testosterone levels. ${ }^{7,8}$ Moreover, seasonal variations in vitamin $\mathrm{D}$ and testosterone levels have also been reported. ${ }^{1,2,9)}$ Our study results support the occurrence of seasonal variations in vitamin $\mathrm{D}$ and total testosterone levels. However, our results do not support a correlation between total testosterone and vitamin D levels, which was reported in previous studies.

During sex hormone synthesis, vitamin D regulates calcium metabolism. ${ }^{12)}$ Moreover, calcium supports male reproductive function, ${ }^{13,14)}$ and the VDR in expressed in reproductive organs such as the testes. Thus, vitamin D could affect testosterone synthesis. Wehr et al. ${ }^{15)} \mathrm{re-}$ ported an association between androgen levels and 25(OH)D levels in men, and many other study findings support the existence of a relationship between vitamin $\mathrm{D}$ and testosterone levels.

In the present study, serum 25(OH)D levels were not correlated with testosterone levels even after adjustment for confounding variables. This result indicates that a different confounding variable, other than the ones considered in this study, could be involved in both vitamin $\mathrm{D}$

Table 2. Seasonal variation in average total testosterone and 25(OH)D levels after adjustment for confounding variables

\begin{tabular}{cccccc}
\hline & \multicolumn{2}{c}{$\begin{array}{c}\text { Correlation between seasonal } \\
\text { variations in total testosterone level }\end{array}$} & & \multicolumn{2}{c}{$\begin{array}{c}\text { Correlation between seasonal } \\
\text { variations in serum 25(OH)D level }\end{array}$} \\
\cline { 5 - 6 } & R squared & P-value & & R squared & P-value \\
\hline Model 1 & - & 0.06 & & $<0.001$ \\
Model 2 & 0.004 & 0.07 & 0.073 & $<0.001$ \\
Model 3 & 0.003 & 0.05 & & 0.095 & $<0.001$ \\
Model 4 & 0.030 & 0.007 & 0.097 & $<0.001$ \\
\hline
\end{tabular}

Calculated by analysis of covariance. Model 1: no adjustment; model 2: adjusted for age; model 3: adjusted for age, total cholesterol, and physical activity; model 4: adjusted for age, total cholesterol, low-density lipoprotein, high-density lipoprotein, waist circumference, body mass index, fasting glucose, physical activity, alcohol use, and smoking.

25(OH)D, 25-hydroxyvitamin D. 
and testosterone metabolism. Tak et al. ${ }^{11)}$ reported that a higher level of 25(OH)D was associated with a higher total testosterone level in Korean men. However, the sample size in their study was different from that in the present study (652 versus 1,559), as were the average laboratory values, such as those of $25(\mathrm{OH}) \mathrm{D}$ and testosterone $(25[\mathrm{OH}] \mathrm{D}$ : 21.23 versus $15.9 \mathrm{ng} / \mathrm{mL}$, testosterone: 4.7 versus $5.1 \mathrm{ng} / \mathrm{mL}$ ). A larger scale study is needed to resolve this discrepancy.

Recent studies that investigated seasonal variations in vitamin D yielded controversial results. Hagenau et al. ${ }^{16)}$ reported that vitamin D levels depend on age, sex, and skin color. However, Oleson et al. ${ }^{17)}$ reported seasonal variations in vitamin D levels in white individuals. This finding was supported by other studies suggesting that vitamin D levels are affected by seasons. ${ }^{2,18)}$ Our results also support the theory that vitamin D levels vary by season, with the highest levels occurring in the summer and the lowest levels in the spring. One of the reasons for this variation could be that during the colder seasons, people wear heavier clothing, which decreases the amount of skin exposed to sunlight, and results in a decrease in vitamin D synthesis. The average temperatures during the spring have decreased in recent years, which could contribute to greater seasonal variation in vitamin D levels.

Similarly, a recent study investigating the effect of seasons on testosterone levels yielded controversial results. González-Sales et al. ${ }^{9)}$ reported that testosterone levels during the winter and spring were higher than the levels during the summer and fall. Svartberg et al. ${ }^{10)}$ reported that testosterone levels were the highest in October and November, and the lowest in June. In contrast, Maes et al. ${ }^{19)}$ and Tancredi et al. ${ }^{20}$ reported that total testosterone and free testosterone levels, respectively, showed no significant seasonal variability.

Our result supports the theory that testosterone levels show seasonal variations, with the highest levels in the spring and the lowest levels in the summer. One possible reason for this variation may be the increased consumption of seafood such as oyster, shrimp, and blue crab during winter and spring (September-February) because of the increased availability of these foods during these seasons. Seafood contains zinc, a trace element known to be important for normal male reproductive function ${ }^{21)}$; thus, this seasonal dietary variation could contribute to the seasonal differences in testosterone levels.

On the basis of our results, we suggest that the reference values of serum 25(OH)D and testosterone levels should be adjusted for seasonal variations after reviewing the results of other studies.

Our study has several limitations. We enrolled subjects who underwent a medical examination at a university hospital. Therefore, our study sample does not reflect the general population, and our findings cannot be generalized. Furthermore, our study has a cross-sectional design, which made it difficult to evaluate prospective changes or the relationships among variables over time. In addition, participants could have self-medicated with vitamin D supplements, which would have significantly affected our results. Another limitation is that, in some men, blood samples were collected in the afternoon, and thus, differences in the time of sample collection could have contributed to differences in total testosterone levels. Finally, the total testosterone level, which was measured in this study, does not represent the level of the active sex hormone.

In conclusion, the serum 25(OH)D level was not correlated with the total testosterone level in Korean men. This result is inconsistent with results of recent studies that found a correlation between vitamin D and testosterone levels; additional studies with a larger general patient population are required to resolve this discrepancy.

In the present study, we found seasonal variations in serum $25(\mathrm{OH})$ $\mathrm{D}$ and total testosterone levels. These associations persisted after adjusting for age, total cholesterol, LDL, HDL, WC, BMI, FG, physical activity, alcohol use, and smoking. Although additional studies are required to confirm these findings, the reference values for vitamin D and testosterone levels in Korean men should be adjusted according to the season during sample collection.

\section{CONFLICT OF INTEREST}

No potential conflict of interest relevant to this article was reported.

\section{REFERENCES}

1. Webb AR. Who, what, where and when-influences on cutaneous vitamin D synthesis. Prog Biophys Mol Biol 2006;92:17-25.

2. Miyauchi M, Hirai C, Nakajima H. The solar exposure time required for vitamin D3 synthesis in the human body estimated by numerical simulation and observation in Japan. J Nutr Sci Vitaminol (Tokyo) 2013;59:257-63.

3. Yeum KJ, Song BC, Joo NS. Impact of geographic location on vitamin D status and bone mineral density. Int J Environ Res Public Health 2016;13:184.

4. Reid IR. What diseases are causally linked to vitamin D deficiency? Arch Dis Child 2016;101:185-9.

5. Blomberg Jensen M. Vitamin D metabolism, sex hormones, and male reproductive function. Reproduction 2012;144:135-52.

6. Pilz S, Frisch S, Koertke H, Kuhn J, Dreier J, Obermayer-Pietsch B, et al. Effect of vitamin D supplementation on testosterone levels in men. Horm Metab Res 2011;43:223-5.

7. Nimptsch K, Platz EA, Willett WC, Giovannucci E. Association between plasma 25-OH vitamin D and testosterone levels in men. Clin Endocrinol (Oxf) 2012;77:106-12.

8. Lunenfeld B, Mskhalaya G, Zitzmann M, Arver S, Kalinchenko S, Tishova $Y$, et al. Recommendations on the diagnosis, treatment and monitoring of hypogonadism in men. Aging Male 2015;18:5-15.

9. Gonzalez-Sales M, Barriere O, Tremblay PO, Nekka F, Desrochers J, Tanguay M. Modeling testosterone circadian rhythm in hypogonadal males: effect of age and circannual variations. AAPS J 2016;18:217-27.

10. Svartberg J, Jorde R, Sundsfjord J, Bonaa KH, Barrett-Connor E. Seasonal variation of testosterone and waist to hip ratio in men: the Tromsø study. J Clin Endocrinol Metab 2003;88:3099-104.

11. Tak YJ, Lee JG, Kim YJ, Park NC, Kim SS, Lee S, et al. Serum 25-hydroxyvitamin D levels and testosterone deficiency in middle-aged Korean men: a cross-sectional study. Asian J Androl 2015;17:324-8.

12. DeLuca HF. Overview of general physiologic features and functions of 
vitamin D. Am J Clin Nutr 2004;80(6 Suppl):1689S-1696S.

13. Bischoff-Ferrari HA, Orav EJ, Dawson-Hughes B. Additive benefit of higher testosterone levels and vitamin D plus calcium supplementation in regard to fall risk reduction among older men and women. Osteoporos Int 2008;19:1307-14.

14. Ayaz O, Howlett SE. Testosterone modulates cardiac contraction and calcium homeostasis: cellular and molecular mechanisms. Biol Sex Differ 2015;6:9.

15. Wehr E, Pilz S, Boehm BO, Marz W, Obermayer-Pietsch B. Association of vitamin D status with serum androgen levels in men. Clin Endocrinol (Oxf) 2010;73:243-8.

16. Hagenau T, Vest R, Gissel TN, Poulsen CS, Erlandsen M, Mosekilde L, et al. Global vitamin D levels in relation to age, gender, skin pigmentation and latitude: an ecologic meta-regression analysis. Osteoporos Int 2009;20:133-40.

17. Oleson CV, Patel PH, Wuermser LA. Influence of season, ethnicity, and chronicity on vitamin D deficiency in traumatic spinal cord injury. J Spinal Cord Med 2010;33:202-13.

18. Alemzadeh R, Kichler J, Babar G, Calhoun M. Hypovitaminosis D in obese children and adolescents: relationship with adiposity, insulin sensitivity, ethnicity, and season. Metabolism 2008;57:183-91.

19. Maes M, Mommen K, Hendrickx D, Peeters D, D'Hondt P, Ranjan R, et al. Components of biological variation, including seasonality, in blood concentrations of TSH, TT3, FT4, PRL, cortisol and testosterone in healthy volunteers. Clin Endocrinol (Oxf) 1997;46:587-98.

20. Tancredi A, Reginster JY, Luyckx F, Legros JJ. No major month to month variation in free testosterone levels in aging males: minor impact on the biological diagnosis of 'andropause.' Psychoneuroendocrinology 2005;30:638-46.

21. Yamaguchi S, Miura C, Kikuchi K, Celino FT, Agusa T, Tanabe S, et al. Zinc is an essential trace element for spermatogenesis. Proc Natl Acad Sci U S A 2009;106:10859-64. 